\title{
Signal Reconstruction Errors in Jittered Sampling
}

\author{
Alessandro Nordio, Member, IEEE, Carla-Fabiana Chiasserini, Senior Member, IEEE, and \\ Emanuele Viterbo, Senior Member, IEEE
}

\begin{abstract}
One of the most significant types of error in digital signal processing (DSP) systems working with wideband signals is the error introduced by the analog-to-digital (AD) and digital-to-analog (DA) converters. This paper presents an accurate and simple method to evaluate the performance of AD/DA converters affected by clock jitter, which is based on the analysis of the mean square error (MSE) between the reconstructed signal and the original one. Using an approximation of the linear minimum MSE (LMMSE) filter as reconstruction technique, we derive analytic expressions of the MSE. In particular, through asymptotic analysis, we are able to simply evaluate the performance of digital signal reconstruction as a function of the clock jitter, number of quantization bits, signal bandwidth and sampling rate.
\end{abstract}

Index Terms-Analog-digital conversion, error analysis, signal reconstruction, signal sampling.

\section{INTRODUCTION}

A SIGNIFICANT problem in analog-digital conversion (ADC) of wideband signals is clock jitter and its impact on the quality of signal reconstruction [i.e., digital-analog conversion (DAC)] [1], [2]. Indeed, even small amounts of jitter can measurably degrade the performance of analog-to-digital (AD) and digital-to-analog (DA) converters; as an example, for a 24-bit quantized audio signal, jitter greater than 3-5 ps can already be extremely harmful.

Clock jitter is typically detrimental because the analog to digital process relies upon a sample clock to indicate when a sample or snap shot of the analog signal is taken. In order to accurately represent the analog data, the sample clock must be evenly spaced in time. Any deviation will result in a distortion of the digitization process since, once an analog signal is converted, it is virtually impossible to recreate the small timing variations in such a way as to reassemble the digital signal back to analog in its original form. If one had a perfect ADC and a perfect DAC and used the same clock to drive both units, then jitter would not have any impact on the reconstructed signal. In a real-world system, however, a digitized signal travels through

Manuscript received October 17, 2008; accepted June 16, 2009. First published July 14, 2009; current version published November 18, 2009. The associate editor coordinating the review of this manuscript and approving it for publication was Dr. Soontorn Oraintara. This work was supported in part by the Regione Piemonte (Italy) through the VICSUM project and in part by the European Commission in the framework of the FP7 Network of Excellence in Wireless COMmunications NEWCOM++ (Contract 216715).

A. Nordio and C.-F. Chiasserini are with the Department of Electronic Engineering, Politecnico di Torino, 10129 Torino, Italy (e-mail: alessandro.nordio@polito.it; carla.chiasserini@polito.it).

E. Viterbo is with the DEIS, Università della Calabria, 87036 Rende (CS), Italy (e-mail: viterbo@deis.unical.it).

Digital Object Identifier 10.1109/TSP.2009.2027404 multiple processors, usually it is stored on a disk or piece of tape for a while, and then goes through more processing before being converted back to analog. Thus, during reconstruction, the clock pulses used to sample the signal are replaced with newer ones with their own subtle variations. Note that, a given amount of clock jitter has a greater effect as the signal amplitude and frequency increase, since in both cases the change in unit time of the signal is greater with high-level, high-frequency signals. Furthermore, depending on the sources, jitter may have different probability distributions, and different probability distributions may have different effects on the quality of the reconstructed signal. In particular, wideband noise generates a randomly distributed jitter and manifests as increased noise and distortion in the signal [3]-[5], hence leading to a decrease in the signal-to-noise ratio (SNR).

While several results are available in the literature on jittered sampling [6], [7] as well as on experimental measurements and instruments performance [3], [5], [8], [9], an analytical methodology for the performance study of the $\mathrm{AD} / \mathrm{DA}$ conversion is still missing.

In this paper we fill this gap and propose a method for evaluating the performance of $\mathrm{AD} / \mathrm{DC}$ converters affected by jitter, which is based on the analysis of the mean square error (MSE) between the reconstructed signal and the original one [9].

The problem of signal reconstruction from irregularly spaced samples (which represent a more general case with respect to jittered samples) has been addressed by several works in the field of signal processing (see, e.g., [10]-[12]), and many reconstruction techniques have been proposed. Here, as reconstruction technique, we consider linear filtering, which has the advantage of enabling a theoretical analysis, unlike other approaches such as iterative or nonlinear techniques. Furthermore, linear filters have been used in a wide variety of fields such as MIMO communication systems [13], multiuser detection [14], and reconstruction of physical fields sampled by sensor networks [15]. In particular, in our previous work [15] we showed that physical fields can be reconstructed with high reliability from an irregularly deployed sensor network whose nodes are characterized by random positions which are known (up to some errors) to the reconstruction algorithm. The analytic approach employed in [15] for deriving the expression of the reconstruction performance is similar to that proposed here for jittered sampling. However, unlike [15], this work deals with regularly spaced samples affected by unknown jitter. This setting leads to a totally different matrix representing the sampling system and to a completely different set of equations and results with respect to those presented in [15]. 
Notice that if jitter is known exactly, the linear minimum MSE (LMMSE) reconstruction technique is optimal in the mean-square sense since it minimizes the MSE of the reconstructed signal. In practice this is not the case, hence we apply a reconstruction filter with the same structure of the LMMSE filter, where we let the jitter vanish. Then, we apply asymptotic analysis to derive analytical expressions of the MSE on the quality of the reconstructed signal. Through numerical results, we show that our asymptotic expressions provide an excellent approximation of the MSE even for small values of the system parameters, with the advantage of greatly reducing the computation complexity. In particular, we look at two different probability distributions of the jitter, namely, Gaussian and uniform distribution, and show that our asymptotic approach provides an excellent approximation of the MSE. Finally, we apply our method to study the performance of the AD/DA conversion system as a function of the clock jitter, number of quantization bits, signal bandwidth and sampling rate.

\section{SYSTEM MODEL}

\section{A. Notations}

Column vectors are denoted by bold lowercase letters and matrices are denoted by bold upper case letters. The $(k, q)$ th entry of the generic matrix $\mathbf{Z}$ is denoted by $(\mathbf{Z})_{k, q}$. The $n \times n$ identity matrix is denoted by $\mathbf{I}_{n}$, while $\mathbf{I}$ is the generic identity matrix. $(\cdot)^{\mathrm{T}}$ is the transpose operator, while $(\cdot)^{\dagger}$ is the conjugate transpose operator. We denote by $f_{x}(z)$ the probability density function (pdf) of the generic random variable $x$, and by $\mathbb{E}[\cdot]$ the average operator.

\section{B. Sampling and Reconstruction Quality}

We consider an analog signal $s(t)$ sampled at constant rate $f_{s}=1 / T_{s}$ over the finite interval $\left[0, M T_{s}\right)$, where $T_{s}$ is the sample spacing. When observed over a finite interval, $s(t)$ admits an infinite Fourier series expansion. Let $N^{\prime}$ denote the largest index of the non-negligible Fourier coefficients, then $N^{\prime} / T_{s}$ can be considered as the approximate one-sided bandwidth of the signal. We therefore represent the signal by using a truncated Fourier series with $N=2 N^{\prime}+1$ complex harmonics:

$$
s(t)=\frac{1}{\sqrt{N}} \sum_{\ell=-N^{\prime}}^{N^{\prime}} a_{\ell} \exp \left(\mathrm{j} 2 \pi \ell \frac{t}{M T_{s}}\right)
$$

$0 \leq t<M T_{s}$. The complex vector $\mathbf{a}=\left[a_{-N^{\prime}}, \ldots, a_{0}\right.$, $\left.\ldots, a_{N^{\prime}}\right]^{\mathrm{T}}$ represents the discrete spectrum of the signal. Observe that the signal representation given in (1) includes sine waves of any fractional frequency $f_{0}=f_{s} N^{\prime} / M$ (when $a_{\ell}=0$ for $-N^{\prime}<\ell<N^{\prime}$ and $a_{-N^{\prime}}=a_{N^{\prime}}^{*}$ ), which are frequently used as reference signal for calibration of ADC [3], [4]. Furthermore, we note that when the signal $s(t)$ is observed in the frequency domain through its $M$ samples, the spectral resolution is given by

$$
\Delta f=\frac{1}{M T_{s}}
$$

Therefore, considering the expression in (1), the signal bandwidth is given by

$$
B=\frac{N \Delta f}{2}=\frac{N}{2 M T_{s}} .
$$

By defining the parameter $\beta$ as

$$
\beta=\frac{M}{N}
$$

we can also write

$$
B=\frac{f_{s} / 2}{\beta}
$$

From (3) it is clear that the parameter $\beta$ represents the oversampling factor of the signal $s(t)$ beyond the Nyquist rate.

In this work, we consider that sampling locations suffer from jitter, i.e., the $m$ th sampling location is given by

$$
t_{m}=m T_{s}+d_{m}, \quad m=0, \ldots, M-1
$$

where $d_{m}$ is the associated independent random jitter whose distribution is denoted by $f_{d}(z)$. Typically, we have $\left|d_{m}\right| \ll T_{s}$.

Let the signal samples be $\mathbf{s}=\left[s_{0}, \ldots, s_{M-1}\right]^{\mathrm{T}}$ where $s_{m}=$ $s\left(t_{m}\right), 0 \leq m \leq M-1$. Using (1), the set of signal samples can be written as

$$
\mathbf{s}=\mathbf{V}^{\dagger} \mathbf{a}
$$

where $\mathbf{V}$ is an $N \times M$ Vandermonde matrix defined as

$$
(\mathbf{V})_{\ell, m}=\frac{1}{\sqrt{N}} \exp \left(-\mathrm{j} 2 \pi \ell \frac{t_{m}}{M T_{s}}\right)
$$

$\ell=-N^{\prime}, \ldots, N^{\prime}$, and $m=0, \ldots, N-1$. Note that $\mathbf{V}$ accounts for the jitter in the AD/DA conversion process, and that the parameter $\beta$ defined in (2) also represents the aspect ratio ${ }^{1}$ of matrix $\mathbf{V}$.

Furthermore, in addition to jittered sampling, we assume that signal samples are affected by some additive noise and are therefore given by

$$
\mathbf{y}=\mathbf{s}+\mathbf{n}
$$

where $\mathbf{n}$ is a vector of $M$ noise samples, modeled as zero mean i.i.d. random variables. In practice, the dominant additive noise error is due to the $n$-bit quantization process [17].

\footnotetext{
${ }^{1}$ The aspect ratio of an $N \times M$ matrix is the ratio between the number of columns and the number of rows.
} 
Now, let us consider a reconstruction technique that provides an estimate $\hat{\mathbf{a}}$ of the discrete spectrum a, and let $\hat{s}(t)$ be the reconstruction of $s(t)$ obtained from $\hat{\mathbf{a}}$, i.e.,

$$
\hat{s}(t)=\frac{1}{\sqrt{N}} \sum_{\ell=-N^{\prime}}^{N^{\prime}} \hat{a}_{\ell} \exp \left(\mathrm{j} 2 \pi \ell \frac{t}{M T_{s}}\right) .
$$

We consider as performance metric of the AD/DA conversion process the mean square error (MSE) associated to the estimate. The MSE, evaluated in the observation interval $\left[0, M T_{s}\right)$, can be equivalently computed in both time and frequency domains as shown below:

$$
\begin{aligned}
& \mathrm{MSE}=\mathbb{E}\left[\int_{0}^{M T_{s}}|s(t)-\hat{s}(t)|^{2} \mathrm{~d} t\right] \\
& =\frac{1}{N} \mathbb{E}\left[\int_{0}^{M T_{s}}\left|\sum_{\ell=-N^{\prime}}^{N^{\prime}} \mathrm{e}^{\mathrm{j} 2 \pi \ell \frac{t}{M T_{s}}}\left(a_{\ell}-\hat{a}_{\ell}\right)\right|^{2} \mathrm{~d} t\right] \\
& =\frac{1}{N} \sum_{\ell, h=-N^{\prime}}^{N^{\prime}} \sum_{h=-N^{\prime}}^{N^{\prime}} \mathbb{E}\left[\left(a_{\ell}-\hat{a}_{\ell}\right)\left(a_{h}-\hat{a}_{h}\right)^{*}\right] \\
& \cdot \int_{0}^{M T_{s}} \mathrm{e}^{\mathrm{j} 2 \pi(\ell-h) \frac{t}{M T_{s}}} \mathrm{~d} t \\
& =\frac{1}{N} \sum_{\ell=-N^{\prime}}^{N^{\prime}} \mathbb{E}\left[\left|a_{\ell}-\hat{a}_{\ell}\right|^{2}\right]=\frac{1}{N} \mathbb{E}\left[\|\mathbf{a}-\hat{\mathbf{a}}\|^{2}\right] .
\end{aligned}
$$

More specifically, we consider as performance metric of the signal reconstruction the MSE relative to the signal average power:

$$
J=\frac{\mathrm{MSE}}{\sigma_{a}^{2}}
$$

which can be thought of as a noise-to-signal ratio and will be plotted in a decibel scale in our results.

Among the possible techniques that can be applied to reconstruct the original signal, we focus on linear filters. Linear filtering provides an estimate of a through the linear operation

$$
\hat{\mathbf{a}}=\mathbf{B y}
$$

where $\mathbf{B}$ is an $N \times M$ matrix. Below, we present the linear reconstruction filter that we apply to the case of jittered ADC/DAC systems.

\section{JitTERED AD/DA CONVERSION With LiNEAR FILTERING}

Let us assume $\|\mathbf{a}\|^{2}=\sigma_{a}^{2} N$ and $\mathbb{E}\left[\mathbf{n n}^{\dagger}\right]=\sigma_{n}^{2} \mathbf{I}$, then we define the SNR in absence of jitter as

$$
\gamma=\frac{\sigma_{a}^{2}}{\sigma_{n}^{2}}
$$

Under the assumption that $\mathbb{E}\left[\mathbf{a a}^{\dagger}\right]=\sigma_{a}^{2} \mathbf{I}$, the linear filter that provides the best performance in terms of MSE is the LMMSE filter, which is given by [14]

$$
\mathbf{B}_{\mathrm{opt}}=\left(\mathbf{V} \mathbf{V}^{\dagger}+\frac{1}{\gamma} \mathbf{I}\right)^{-1} \mathbf{V}
$$

In [15], it has been shown that, by applying the LMMSE, we obtain

$$
J=\frac{1}{\sigma_{a}^{2} N} \mathbb{E}\left[\|\mathbf{a}-\hat{\mathbf{a}}\|^{2}\right]=\mathbb{E}\left[\operatorname{tr}\left\{\left(\gamma \mathbf{V} \mathbf{V}^{\dagger}+\mathbf{I}\right)^{-1}\right\}\right]
$$

where $\operatorname{tr}\{\cdot\}$ is the normalized matrix trace operator.

Note, however, that the filter in (7) cannot be employed in practice, since the jitters $d_{m}$ (hence the matrix $\mathbf{V}$ ) are unknown [see the definition of $\mathbf{V}$ in (5)]. We therefore resort to an approximation of the optimum filter $\mathbf{B}_{\text {opt }}$, based on the assumption that jitter has a zero mean.

In particular, we approximate $\mathbf{V}$ with the matrix $\mathbf{F}$ defined as

$$
\mathbf{F}=\left.\mathbf{V}\right|_{d_{m}=0}
$$

with the generic element of $\mathbf{F}$ given by

$$
(\mathbf{F})_{\ell, m}=\frac{1}{\sqrt{N}} \exp \left(-\mathrm{j} 2 \pi \ell \frac{m}{M}\right)
$$

$\ell=-N^{\prime}, \ldots, N^{\prime}$, and $m=0, \ldots, N-1$. We observe that $\mathbf{F}$ has the following property:

$$
\mathbf{F F}^{\dagger}=\beta \mathbf{I}
$$

and it is related to the discrete Fourier transform matrix.

Substituting the approximation of $\mathbf{V}$ in (7), we obtain:

$$
\mathbf{B}=\left(\beta+\frac{1}{\gamma}\right)^{-1} \mathbf{F} .
$$

Notice that the filter in (8) is the LMMSE filter adapted to the linear model $\mathbf{y}=\mathbf{F}^{\dagger} \mathbf{a}+\mathbf{n}$. By letting $\omega=(\beta+1 / \gamma)^{-1}$, the noise to signal ratio $J$ provided by the approximate filter (8) is given by

$$
\begin{aligned}
J & =\frac{1}{\sigma_{a}^{2} N} \mathbb{E}\left[\|\mathbf{a}-\omega \mathbf{F} \mathbf{y}\|^{2}\right] \\
& =\frac{1}{\sigma_{a}^{2} N} \mathbb{E}\left[\left\|\left(\omega \mathbf{F} \mathbf{V}^{\dagger}-\mathbf{I}\right) \mathbf{a}+\omega \mathbf{F} \mathbf{n}\right\|^{2}\right] \\
& =\operatorname{tr}\left\{\mathbb{E}_{d}\left[\left(\omega \mathbf{F} \mathbf{V}^{\dagger}-\mathbf{I}\right)\left(\omega \mathbf{V} \mathbf{F}^{\dagger}-\mathbf{I}\right)\right]+\frac{\omega^{2} \beta}{\gamma} \mathbf{I}\right\} \\
& =\operatorname{tr}\left\{\omega^{2} \mathbb{E}_{d}\left[\mathbf{F} \mathbf{V}^{\dagger} \mathbf{V} \mathbf{F}^{\dagger}\right]-2 \omega \Re \mathbb{E}_{d}\left[\mathbf{F} \mathbf{V}^{\dagger}\right]+\frac{\gamma+\omega^{2} \beta}{\gamma} \mathbf{I}\right\}
\end{aligned}
$$

where the operator $\mathbb{E}_{d}[\cdot]$ averages over the random jitters $d_{m}, m=0, \ldots, M-1$.

Assuming that the jitters are independent [3] and considering that the jitter characteristic function is $C_{d}(w)=\mathbb{E}_{d}[\exp (\mathrm{j} w z)]$, 
in Appendix A we derive the following expressions for the two terms in (9):

$$
\begin{aligned}
& \operatorname{tr}\left\{\mathbb{E}_{d}\left[\mathbf{F} \mathbf{V}^{\dagger}\right]\right\}=\frac{\beta}{N} \sum_{\ell=-N^{\prime}}^{N^{\prime}} C_{d}\left(\frac{2 \pi \ell}{M T_{s}}\right) \\
& \operatorname{tr}\left\{\mathbb{E}_{d}\left[\mathbf{F} \mathbf{V}^{\dagger} \mathbf{V} \mathbf{F}^{\dagger}\right]\right\} \\
& =\beta+\beta \frac{(\beta-1)}{N} \sum_{\ell=-N^{\prime}}^{N^{\prime}}\left|C_{d}\left(\frac{2 \pi \ell}{M T_{s}}\right)\right|^{2} .
\end{aligned}
$$

Hence, we write the noise to signal ratio $J$ as

$$
\begin{array}{r}
J=1+\omega^{2} \beta\left(1+\frac{1}{\gamma}\right)-2 \omega \frac{\beta}{N} \sum_{\ell=-N^{\prime}}^{N^{\prime}} C_{d}\left(\frac{2 \pi \ell}{M T_{s}}\right) \\
+\omega^{2} \beta \frac{(\beta-1)}{N} \sum_{\ell=-N^{\prime}}^{N^{\prime}}\left|C_{d}\left(\frac{2 \pi \ell}{M T_{s}}\right)\right|^{2} .
\end{array}
$$

In order to reduce the complexity of the computation of the reconstruction error and provide simple but accurate analytical tools, in the next section we let the parameters $N$ and $M$ go to infinity, while the ratio $\beta=M / N$ is kept constant. We therefore derive an asymptotic expression of $J$, which we will show to well approximate the expression in (12).

\section{ASYMPTOTIC ANALYSIS}

When the parameters $N$ and $M$ grow to infinity while $\beta$ is kept constant, we define the asymptotic noise-to-signal ratio $J$ as

$$
\mathrm{J}_{\infty}^{(\beta, \gamma)}=\lim _{N, M \rightarrow+\infty} J
$$

In [15], it has been shown that $\mathrm{J}_{\infty}^{(\beta, \gamma)}$ provides an excellent approximation of $\mathrm{MSE} / \sigma_{a}^{2}$ even for small values of $N$ and $M$, with the advantage of greatly simplifying the computation.

In the limit $N, M \rightarrow \infty$ with constant $\beta$, we compute

$$
\begin{aligned}
\mu_{1} & =\lim _{N, M \rightarrow+\infty} \frac{1}{N} \sum_{\ell=-N^{\prime}}^{N^{\prime}} C_{d}\left(\frac{2 \pi \ell}{M T_{s}}\right) \\
& =\lim _{N, M \rightarrow+\infty} \frac{1}{N} \sum_{\ell=-N^{\prime}}^{N^{\prime}} C_{d}\left(\frac{2 \pi \ell}{\beta N T_{s}}\right) \\
& =\int_{-1 / 2}^{1 / 2} C_{d}\left(\frac{2 \pi x}{\beta T_{s}}\right) \mathrm{d} x \\
& =\int_{-1 / 2}^{1 / 2} C_{d}(4 \pi B x) \mathrm{d} x .
\end{aligned}
$$

where, from (3), we used the fact that $1 / \beta T_{s}=f_{s} / \beta=2 B$. Similarly, we define

$$
\begin{aligned}
\mu_{2} & =\lim _{N, M \rightarrow+\infty} \frac{1}{N} \sum_{\ell=-N^{\prime}}^{N^{\prime}} C_{d}\left(\frac{2 \pi \ell}{M T_{s}}\right)^{2} \\
& =\int_{-1 / 2}^{1 / 2}\left|C_{d}(4 \pi B x)\right|^{2} \mathrm{~d} x .
\end{aligned}
$$

By using (13) (14), and (12), the asymptotic expression of $J$ is given by

$$
\mathrm{J}_{\infty}^{(\beta, \gamma)}=1+\omega^{2} \beta(1+1 / \gamma)-2 \omega \beta \mu_{1}+\omega^{2} \beta(\beta-1) \mu_{2} .
$$

It is worth mentioning that for large SNRs (i.e., in absence of measurement noise), $\mathrm{J}_{\infty}^{(\beta, \gamma)}$ reduces to

$$
\mathrm{J}_{\infty}^{(\beta)}=\lim _{\gamma \rightarrow \infty} \mathrm{J}_{\infty}^{(\beta, \gamma)}=1+\frac{1}{\beta}-2 \mu_{1}+\left(1-\frac{1}{\beta}\right) \mu_{2} .
$$

By also letting $\beta$ go to infinity, i.e., for highly oversampled signals, $\mathrm{J}_{\infty}^{(\beta)}$ reduces to

$$
\mathrm{J}_{\infty}=\lim _{\beta \rightarrow \infty} \mathrm{J}_{\infty}^{(\beta)}=1-2 \mu_{1}+\mu_{2}
$$

Equations (16) and (17) provide us with two floor values that represent the best quality of the reconstructed signal (minimum MSE) we can hope for.

Below we present examples for two jitter probability distributions, namely, Gaussian and uniform, which are often assumed to characterize the jitter affecting AD/DA converters.

\section{A. Gaussian Jitter Distribution}

If jitter is assumed to follow a Gaussian distribution with variance $\sigma^{2}$ [8], then the characteristic function $C_{d}(w)$ is given by

$$
C_{d}(w)=\exp \left(-\frac{1}{2} \sigma^{2} w^{2}\right)
$$

By using (13) and (14), we obtain

$$
\begin{aligned}
& \mu_{1}=\frac{1}{\sqrt{8 \pi} \eta_{g}} \operatorname{erf}\left(\sqrt{2} \pi \eta_{g}\right) \\
& \mu_{2}=\frac{1}{4 \sqrt{\pi} \eta_{g}} \operatorname{erf}\left(2 \pi \eta_{g}\right)
\end{aligned}
$$

where $\eta_{g}=\sigma B$ is a dimensionless parameter which relates jitter standard deviation and signal bandwidth. The asymptotic value of $J$ in (15) therefore becomes

$$
\begin{aligned}
\mathrm{J}_{\infty}^{(\beta, \gamma)}=1+\omega^{2} \beta(1+1 / \gamma)-2 \omega \beta & \frac{\operatorname{erf}\left(\sqrt{2} \pi \eta_{g}\right)}{\sqrt{8 \pi} \eta_{g}} \\
& +\omega^{2} \beta(\beta-1) \frac{\operatorname{erf}\left(2 \pi \eta_{g}\right)}{4 \sqrt{\pi} \eta_{g}}
\end{aligned}
$$

however, for the ease of computation, when $\eta_{g} \ll 1$ (i.e., $\sigma \ll$ $1 / B)$, it can be written using its Taylor expansion, which is given by

$$
\mathrm{J}_{\infty}^{(\beta, \gamma)}=\frac{1}{\beta \gamma+1}+\frac{4 \pi^{2} \gamma \beta(\gamma+1)}{3(\beta \gamma+1)^{2}} \eta_{g}^{2}+o\left(\eta_{g}^{4}\right) .
$$

\section{B. Uniform Jitter Distribution}

Let us now assume the jitter to be uniformly distributed with pdf given by

$$
f_{d}(z)= \begin{cases}\frac{1}{2 d_{\max }}, & -d_{\max } \leq z \leq d_{\max } \\ 0, & \text { elsewhere }\end{cases}
$$


where $d_{\max }$ is the maximum jitter, independent of the sampling frequency $f_{s}$. In this case, the characteristic function of the jitter is given by

$$
C_{d}(w)=\frac{\sin \left(d_{\max } w\right)}{d_{\max } w} .
$$

Then, we can write the parameters $\mu_{1}$ and $\mu_{2}$ as

$$
\begin{aligned}
& \mu_{1}=\frac{\operatorname{Si}\left(2 \pi \eta_{u}\right)}{2 \pi \eta_{u}} \\
& \mu_{2}=\frac{\cos ^{2}\left(2 \pi \eta_{u}\right)+2 \pi \eta_{u} \operatorname{Si}\left(4 \pi \eta_{u}\right)-1}{4 \pi^{2} \eta_{u}^{2}}
\end{aligned}
$$

where $\operatorname{Si}(\cdot)$ is the integral sine function and $\eta_{u}=d_{\max } B$ is a dimensionless parameter which relates maximum jitter and signal bandwidth. The asymptotic value of $J$ in (15) therefore becomes

$$
\begin{aligned}
\mathrm{J}_{\infty}^{(\beta, \gamma)}=1+\omega^{2} \beta(1+1 / \gamma)-2 \omega \beta \frac{\operatorname{Si}\left(2 \pi \eta_{u}\right)}{2 \pi \eta_{u}} \\
+\omega^{2} \beta(\beta-1) \frac{\cos ^{2}(2 \pi \eta)+2 \pi \eta_{u} \operatorname{Si}\left(4 \pi \eta_{u}\right)-1}{4 \pi^{2} \eta_{u}^{2}}
\end{aligned}
$$

which, when $\eta_{u} \ll 1$ (i.e., $d_{\max } \ll 1 / B$ ), can be written using its Taylor expansion as

$$
\mathrm{J}_{\infty}^{(\beta, \gamma)}=\frac{1}{\beta \gamma+1}+\frac{4 \pi^{2} \gamma \beta(\gamma+1)}{9(\beta \gamma+1)^{2}} \eta_{u}^{2}+o\left(\eta_{u}^{4}\right) .
$$

Notice that the variance of the uniformly distributed jitter is given by $d_{\max }^{2} / 3$, while the variance of the Gaussian jitter is $\sigma^{2}$. When the two variances are equal (i.e., $\sigma^{2}=d_{\max }^{2} / 3$, which implies $\eta_{u}^{2}=\eta_{g}^{2} / 3$ ), the expressions of $\mathrm{J}_{\infty}^{(\beta, \gamma)}$ in (18) and in (19) are equivalent. This suggests that the reconstruction error depends asymptotically on the jitter variance rather than on the jitter distribution.

In the next section we show that these approximations are extremely accurate, even for $\eta_{g}, \eta_{u}$ of the order of $10^{-1}$.

\section{RESULTS}

Here, we exploit the expressions we derived in the previous sections to study the performance of the AD/DA conversion as the system parameters vary. As already pointed out in Section IV-B, Gaussian and uniform jitter distributions provide very similar performance in terms of $\mathrm{J}_{\infty}^{(\beta, \gamma)}$, thus in the following we present numerical results only for the case of uniformly distributed jitter.

For the ease of representation, we assume that the dominant component of the additive noise is due to quantization, and we express the SNR in absence of jitter $\gamma$ as a function of the number of quantization bits $n$ of the ADC [16],

$$
(\gamma)_{\mathrm{dB}}=6.02 n+1.76 .
$$

Then, in the following plots we show the value of $J$ as a function of $\gamma$ or, equivalently, of the number of quantization bits $n$.

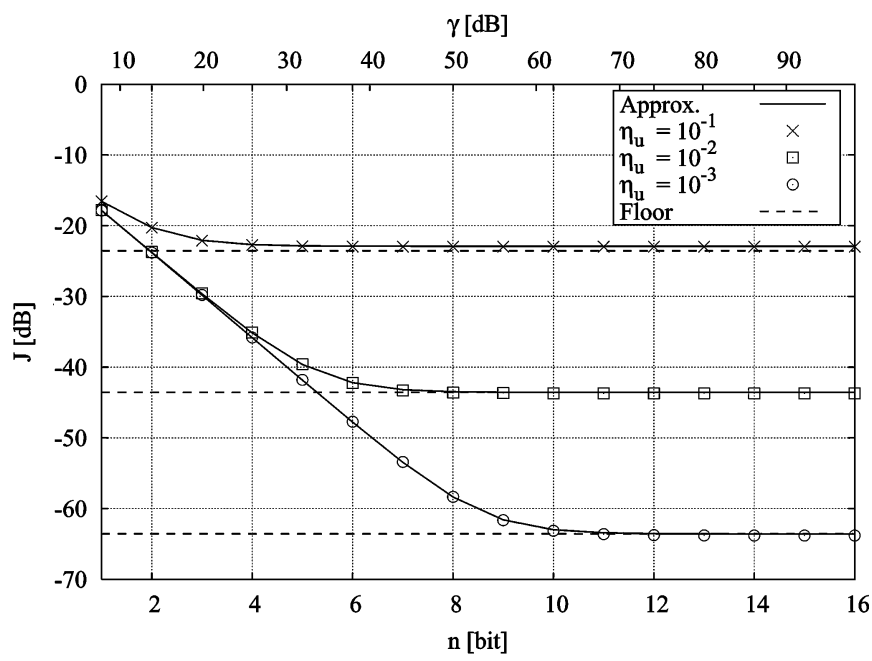

Fig. 1. Comparison between the reconstruction error $J$ derived through (12), the approximation derived through (19) and the floor $\mathrm{J}_{\infty}^{(\beta)}$ in (20).

\section{A. Validity of the Asymptotic Analysis}

We first assess the validity of the asymptotic expression in (19) as an approximation of the reconstruction performance metric in (12).

In Fig. 1, we compare the approximation obtained through (19) (represented by solid lines) against the values of $J$ computed through (12) (represented by markers), for $N^{\prime}=100$. The results are derived for $\eta_{u}=10^{-1}, 10^{-2}, 10^{-3}$, and $\beta=10$. We notice that $J$, when expressed in decibels, first decreases linearly with $n$, then, after a sharp transition, it shows a floor whose expression is given by (16). In the case of uniform jitter distribution, for $\beta>1$ and $\eta_{u} \ll 1$, the floor expression in (16) can be written through its Taylor expansion, as

$$
\mathrm{J}_{\infty}^{(\beta)}=\frac{4 \pi^{2}}{9 \beta} \eta_{u}^{2}+\frac{4 \pi^{4}(5 \beta-8)}{225 \beta} \eta_{u}^{4}+o\left(\eta_{u}^{4}\right) .
$$

In Fig. 1 the floors, computed through the approximated formula in (20), are indicated with the dashed lines.

In general we observe an excellent matching between the approximation computed through (19) and the results computed through (12), even for small values of $N$ and $M$. We point out that this tight match can be observed for any $\beta>1$ and $\eta_{u} \ll 1$, and extends to the floor values. This suggests that the asymptotic expression in (19) can be considered instead of $J$, for evaluating the performance of the $\mathrm{A} / \mathrm{D}$ and $\mathrm{D} / \mathrm{A}$ converters; thus, from now on, we will use the expression given in (19).

\section{B. On the Floor of $J$}

We now focus on the floor of $\mathrm{J}_{\infty}^{(\beta, \gamma)}$ (i.e., of $J$ ) and give an explanation for that. We first notice that the expression in (20) decreases with the oversampling factor $\beta$ and is lower-bounded by

$$
\mathrm{J}_{\infty}=\lim _{\beta \rightarrow \infty} \mathrm{J}_{\infty}^{(\beta)}=\frac{4 \pi^{4}}{45} \eta_{u}^{4}+o\left(\eta_{u}^{4}\right)
$$




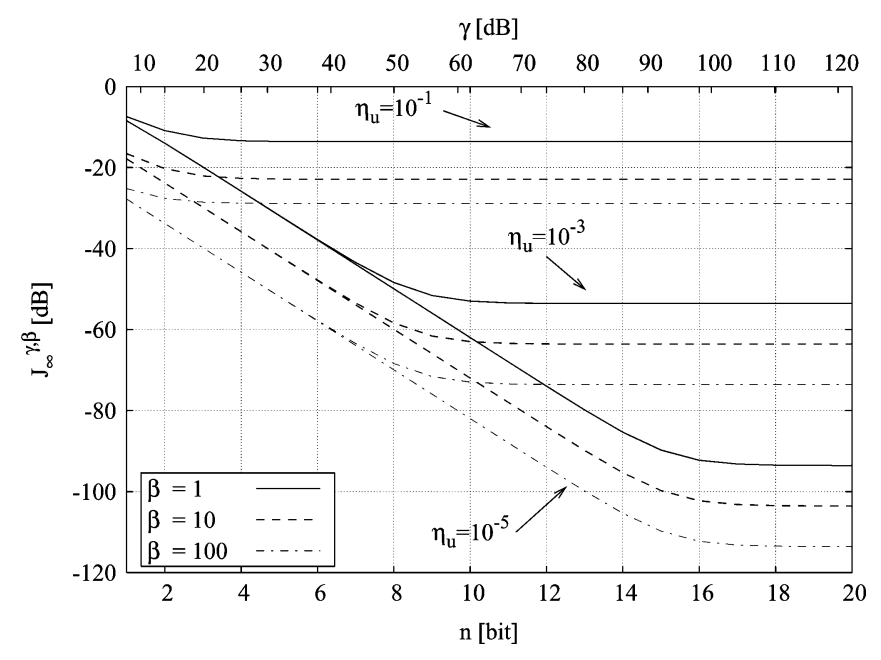

Fig. 2. Approximated $\mathrm{J}_{\infty}^{(\beta, \gamma)}$ obtained through (19) as a function of the ADC number of quantization bits $n$.

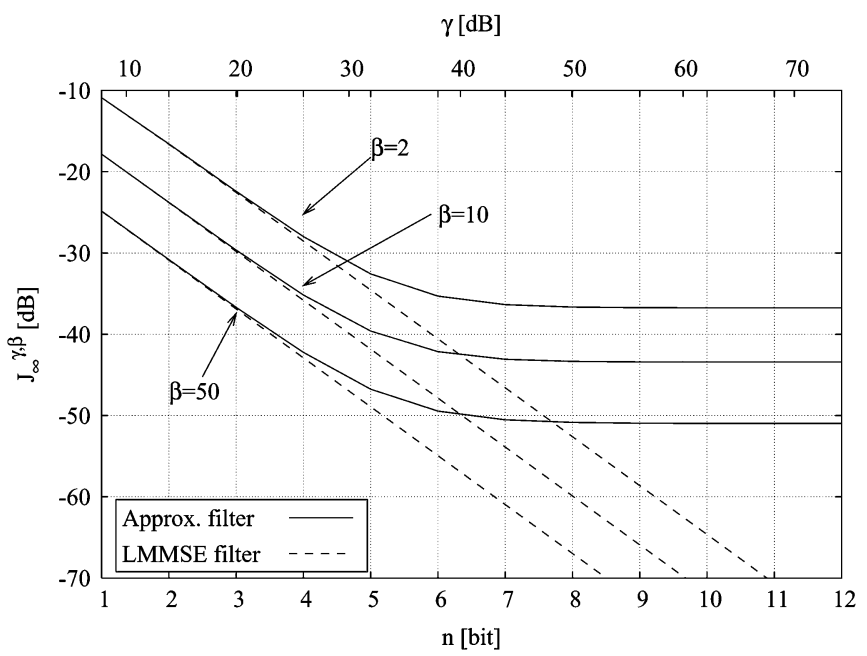

Fig. 3. Comparison of reconstruction performance obtained through the optimal LMMSE filter (7) and the approximated filter (8).

This behavior is confirmed by the results in Fig. 2 where we can appreciate the effect of an increasing $\beta$ for $\eta_{u}=10^{-1}, 10^{-3}, 10^{-5}$.

Then, it is interesting to note that the presence of the floor observed in Figs. 1 and 2 for large values of $\gamma$ is due to the mismatch between the matrix $\mathbf{F}$ employed in the reconstruction and the matrix $\mathbf{V}$ characterizing the sampling system. Indeed, if the jitter were known, we could have used the LMMSE filter $\mathbf{B}_{\text {opt }}$ in (7) instead of the filter $\mathbf{B}$ in (8) for reconstructing the signal: by using the LMMSE filter, the reconstruction error would decrease monotonically as $\gamma$ decreases. The comparison between the two filters is shown in Fig. 3, for $\beta=2,10,50$ and $\eta_{u}=10^{-2}$; there the performance of the LMMSE filter has been derived by considering the values of the jitter to be known, which is not the case in the practice.

\section{Optimal Number of Quantization Bits}

In the case of unknown jitter, and, thus, in the presence of a floor in the behavior of $J$, there exists a number of quantization

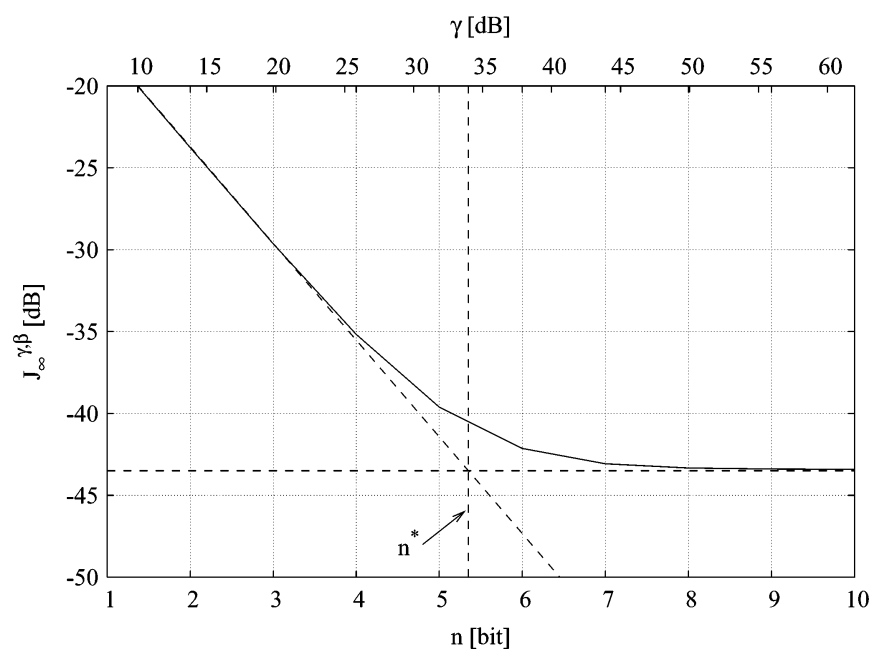

Fig. 4. Estimate of the minimum $n$ required to reach the reconstruction error floor, for $\beta=10$ and $\eta_{u}=10^{-2}$.

bits $n=n^{*}$ beyond which a further increase in the ADC precision does not provide a noticeable decrease in the reconstruction error $J$.

For any given $\beta$ and $\eta_{u}$, the value of $n^{*}$ can be estimated as shown in Fig. 4, where the reconstruction error is plotted versus $\gamma$ (solid line). The horizontal dashed line represents the approximated error floor as in (20), while the dashed line tangent to the reconstruction error in $n=1$ represents a first-order approximation of $J$ for low values of $n$. The intersection of the two lines identifies $n^{*}$, i.e., the minimum $n$ required at the $\mathrm{ADC}$ to reach the reconstruction error floor.

We apply the method described in Fig. 4 for $\eta_{u}$ in the range $\left\{10^{-8}, 10^{-2}\right\}$, and for $\beta=1,2,5,10,100$. The resulting values of $n^{*}$ are shown in Fig. 5. Note that $n^{*}$ is slightly affected by an increase in $\beta$, provided that $\beta>1$, and a good compromise for choosing the oversampling rate is $\beta=5$.

These results can provide useful insights to system designers, as highlighted in the following examples.

Example 1: Consider an ADC with $n=8$ quantization bits, which samples a signal of bandwidth $B=100 \mathrm{MHz}$. The ADC is affected by a jitter whose maximum value is $d_{\max }=10 \mathrm{ps}$. We are interested in determining the sampling rate so that $J \leq$ $55 \mathrm{~dB}$. Since $\eta_{u}=d_{\max } B=10^{-3}$, by looking at Fig. 2 we observe that it is sufficient to have an oversampling ratio $\beta \geq 10$ (i.e., $f_{s} \geq 1 \mathrm{GHz}$ ).

Example 2: An ADC samples a signal of bandwidth $B=1$ $\mathrm{MHz}$, with rate $f_{s}=100 \mathrm{MHz}$ (i.e., $\beta=100$ ). Thus, when the maximum jitter is $d_{\max }=50 \mathrm{ps}$, we have $\eta_{u}=5 \cdot 10^{-5}$, and from Fig. 5 we observe that $n^{*}=13$ is sufficient to reach the reconstruction error floor. When instead $d_{\max }=1$ ps (i.e., $\eta_{u}=10^{-6}$ ), then at least 19 quantization bits are required to achieve the error floor.

\section{CONCLUSION}

We studied the performance of analog-to-digital and digital-to-analog converters, in presence of clock jitter and quantization errors. We considered that a linear filter approximating the LMMSE filter is used for signal reconstruction, and evaluated the system performance in terms of minimum square error 


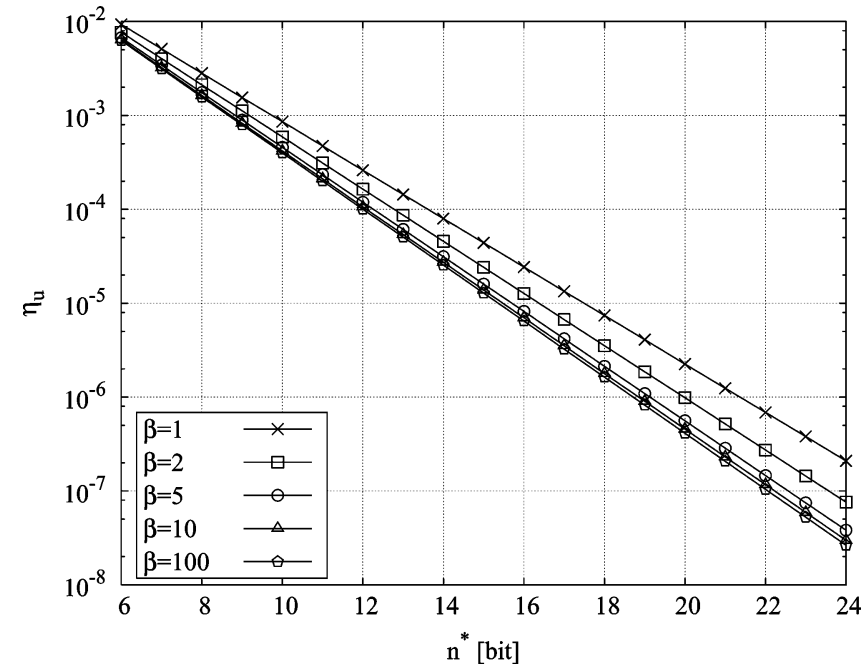

Fig. 5. Minimum number of bits $n^{*}$ required to reach the floor of $\mathrm{J}_{\infty}^{(\beta, \gamma)}$ as a function of $\beta$ and $\eta_{u}$.

between the reconstructed signal and the original one. Through asymptotic analysis, we derived analytical expressions of the MSE which provide an accurate and simple method to evaluate the behavior of AD/DA converters as clock jitter, number of quantization bits, signal bandwidth and sampling rate vary. In particular, we looked at two different probability distributions of the jitter, namely, Gaussian and uniform distribution, and we showed that our asymptotic approach provides an excellent approximation of the MSE even for small values of the system parameters. Furthermore, we derived the MSE floor, which represents the best reconstruction quality level we can hope for and gives useful insights for the design of AD/DA converters.

\section{APPENDIX A}

PROOF OF (10) AND (11)

To derive (10) and (11), first recall the expression in (4), from which we notice that the ratio $t_{m} /\left(M T_{s}\right)$ that appears in (5) is given by

$$
\frac{t_{m}}{M T_{s}}=\frac{m}{M}+\frac{d_{m}}{M T_{s}}
$$

Therefore, we obtain

$$
\begin{aligned}
\operatorname{tr}\left\{\mathbb{E}_{d}\left[\mathbf{F V}^{\dagger}\right]\right\} & =\frac{1}{N^{2}} \mathbb{E}_{d}\left[\sum_{\ell=-N^{\prime}}^{N^{\prime}} \sum_{m=0}^{M-1} \mathrm{e}^{-\mathrm{j} 2 \pi \ell m / M} \mathrm{e}^{+\mathrm{j} 2 \pi \ell\left(m+d_{m} / T_{s}\right) / M}\right] \\
& =\frac{1}{N^{2}} \mathbb{E}_{d}\left[\sum_{\ell=-N^{\prime}}^{N^{\prime}} \sum_{m=0}^{M-1} \mathrm{e}^{+\mathrm{j} 2 \pi \ell \frac{d_{m}}{M T_{s}}}\right] \\
& =\frac{1}{N^{2}} \sum_{\ell=-N^{\prime}}^{N^{\prime}} \sum_{m=0}^{M-1} \mathbb{E}_{d}\left[\mathrm{e}^{+\mathrm{j} 2 \pi \ell \frac{d_{m}}{M T_{s}}}\right] .
\end{aligned}
$$

By defining

$$
C_{d}(w)=\mathbb{E}_{d}[\exp (\mathrm{j} w z)]=\int \exp (\mathrm{j} w z) f_{d}(z) \mathrm{d} z
$$

as the characteristic function of the jitter $d$, we observe that $\mathbb{E}_{d}\left[\mathrm{e}^{+\mathrm{j} 2 \pi \ell\left(d_{m}\right) /\left(M T_{s}\right)}\right]=C_{d}\left((2 \pi \ell) /\left(M T_{s}\right)\right)$. Therefore,

$$
\begin{aligned}
\operatorname{tr}\left\{\mathbb{E}_{d}\left[\mathbf{F} \mathbf{V}^{\dagger}\right]\right\} & =\frac{1}{N^{2}} \sum_{\ell=-N^{\prime}}^{N^{\prime}} \sum_{m=0}^{M-1} C_{d}\left(\frac{2 \pi \ell}{M T_{s}}\right) \\
& =\frac{M}{N^{2}} \sum_{\ell=-N^{\prime}}^{N^{\prime}} C_{d}\left(\frac{2 \pi \ell}{M T_{s}}\right) .
\end{aligned}
$$

Similarly, we write

$$
\begin{aligned}
& \operatorname{tr}\left\{\mathbb{E}_{d}\left[\mathbf{F} \mathbf{V}^{\dagger} \mathbf{V} \mathbf{F}^{\dagger}\right]\right\}=\frac{1}{N^{3}} \mathbb{E}_{d}\left[\sum_{\ell, \ell^{\prime}, m, m^{\prime}} \mathrm{e}^{-\mathrm{j} 2 \pi \ell m / M} \mathrm{e}^{+\mathrm{j} 2 \pi \ell^{\prime}\left(m+d_{m} / T_{s}\right) / M}\right. \\
&\left.\cdot \mathrm{e}^{-\mathrm{j} 2 \pi \ell^{\prime}\left(m^{\prime}+d_{m^{\prime}} / T_{s}\right) / M} \mathrm{e}^{\mathrm{j} 2 \pi \ell m^{\prime} / M}\right] \\
&=\frac{1}{N^{3}} \mathbb{E}_{d}\left[\sum_{\ell, \ell^{\prime}, m, m^{\prime}} \mathrm{e}^{-\mathrm{j} 2 \pi\left(\ell-\ell^{\prime}\right)\left(m-m^{\prime}\right) / M}\right] \\
&\left.\cdot \mathrm{e}^{+\mathrm{j} 2 \pi \ell^{\prime}\left(d_{m}-d_{m^{\prime}}\right) /\left(M T_{s}\right)}\right] \\
&=L_{1}+L_{2}
\end{aligned}
$$

where $L_{1}$ and $L_{2}$ are the contributions to (23) when $m=m^{\prime}$ and $m \neq m^{\prime}$, respectively. Thus, when $m=m^{\prime}$, we have

$$
L_{1}=\frac{1}{N^{3}} \mathbb{E}_{d}\left[\sum_{\ell, \ell^{\prime}, m, m^{\prime}} 1\right]=\frac{M}{N}
$$

while when $m \neq m^{\prime}$ we have

$$
\begin{aligned}
L_{2}= & \frac{1}{N^{3}} \sum_{\ell, \ell^{\prime}, m} \sum_{m^{\prime} \neq m} \mathrm{e}^{-\mathrm{j} 2 \pi\left(\ell-\ell^{\prime}\right)\left(m-m^{\prime}\right) / M} \\
& \cdot C_{d}\left(\frac{2 \pi \ell^{\prime}}{M T_{s}}\right) C_{d}\left(-\frac{2 \pi \ell^{\prime}}{M T_{s}}\right) \\
= & \frac{1}{N^{3}} \sum_{\ell, \ell^{\prime}, m} \sum_{m^{\prime} \neq m} \mathrm{e}^{-\mathrm{j} 2 \pi\left(\ell-\ell^{\prime}\right)\left(m-m^{\prime}\right) / M}\left|C_{d}\left(\frac{2 \pi \ell^{\prime}}{M T_{s}}\right)\right|^{2} \\
= & L_{3}+L_{4}
\end{aligned}
$$

where $L_{3}$ and $L_{4}$ are the contributions to (24) when $\ell=\ell^{\prime}$ and $\ell \neq \ell^{\prime}$, respectively. We obtain

$$
\begin{aligned}
L_{3} & =\frac{M(M-1)}{N^{3}} \sum_{\ell^{\prime}}\left|C_{d}\left(\frac{2 \pi \ell^{\prime}}{M T_{s}}\right)\right|^{2} \\
L_{4} & =-\frac{M}{N^{3}} \sum_{\ell} \sum_{\ell^{\prime} \neq \ell}\left|C_{d}\left(\frac{2 \pi \ell^{\prime}}{M T_{s}}\right)\right| \\
& =-\frac{M(N-1)}{N^{3}} \sum_{\ell}\left|C_{d}\left(\frac{2 \pi \ell}{M T_{s}}\right)\right|^{2} .
\end{aligned}
$$


In conclusion, we get

$\operatorname{tr}\left\{\mathbb{E}_{d}\left[\mathbf{F} \mathbf{V}^{\dagger} \mathbf{V F}{ }^{\dagger}\right]\right\}=\frac{M}{N}+\frac{M(M-N)}{N^{3}} \sum_{\ell}\left|C_{d}\left(\frac{2 \pi \ell}{M T_{s}}\right)\right|^{2}$.

\section{ACKNOWLEDGMENT}

The authors are grateful for the inspiring discussions with Prof. D. Grimaldi, Prof. L. Michaeli, and Dr. M. Ortolano about the many practical aspects of analog-to-digital and digital-toanalog converters.

\section{REFERENCES}

[1] M. Shinagawa et al., "Jitter analysis of high-speed sampling systems," IEEE J. Solid-State Circuits, vol. 25, pp. 220-224, Feb. 1990.

[2] G. D. Muginov and A. N. Venetsanopoulos, "Evaluation of analog to digital conversion error for wideband signals," presented at the IEEE Instrumentation Measurement Technology Conf., Brussels, Belgium, Jun. 1996.

[3] Project DYNAD, SMT4-CT98, Draft Standard Version 3.4, , Jul. 2001.

[4] IEEE Standard for Terminology and Test Methods for Analog-to-Digital Converters, IEEE Std. 1241, 2000.

[5] P. Arpaia, P. Daponte, and S. Rapuano, "Characterization of digitizer timebase jitter by means of the Allan variance," Comput. Stand. Interfac., vol. 25, pp. 15-22, 2003.

[6] B. Liu and T. P. Stanley, "Error bounds for jittered sampling," IEEE Trans. Autom. Control, vol. 10, no. 4, pp. 449-454, Oct. 1965.

[7] J. Tourabaly and A. Osseiran, "A jittered-sampling correction technique for ADCs," in Proc. IEEE Int. Workshop Electronic Design, Test, Applications, Los Alamitos, CA, 2008, pp. 249-252.

[8] E. Rubiola, A. Del Casale, and A. De Marchi, "Noise induced time interval measurement biases," in Proc. 46th IEEE Frequency Control Symp., May 1992, pp. 265-269.

[9] J. Verspecht, "Accurate spectral estimation based on measurements with a distorted-timebase digitizer," IEEE Trans. Instrum. Meas., vol. 43, pp. 210-215, Apr. 1994.

[10] H. G. Feichtinger, K. Gröchenig, and T. Strohmer, "Efficient numerical methods in non-uniform sampling theory," Numer. Math., vol. 69, pp. 423-440, 1995.

[11] F. A. Marvasti, Nonuniform Sampling: Theory and Practice. New York: Kluwer, 2001.

[12] H. Rauhut, Random Sampling of Sparse Trigonometric Polynomials. [Online]. Available: http://homepage.univie.ac.at/holger.rauhut/RandomSampling.pdf

[13] A. Nordio and G. Taricco, "Linear receivers for the multiple-input multiple-output multiple access channel," IEEE Trans. Commun., vol. 54, no. 8, pp. 1446-1456, Aug. 2006.

[14] S. Verdù, Multiuser Detection. Cambridge, U.K.: Cambridge Univ. Press, 1998

[15] A. Nordio, C.-F. Chiasserini, and E. Viterbo, "Performance of linear field reconstruction techniques with noise and uncertain sensor locations," IEEE Trans. Signal Process., vol. 56, no. 8, pp. 3535-3547, Aug. 2008.

[16] G. Gielen, "Analog building blocks for signal processing," ESAT-MICAS, Leuven, Belgium, 2006.

[17] S. C. Ergen and P. Varaiya, "Effects of A-D conversion nonidealities on distributed sampling in dense sensor networks," presented at the 5th Int. Symp. Information Processing Sensor Networks (IPSN), Nashville, Tennessee, Apr. 2006.

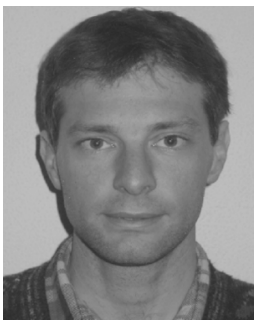

Alessandro Nordio (S'00-M'03) was born in Susa, Italy, in 1974. He received the Laurea degree in telecommunications engineering from Politecnico di Torino, Italy, in 1998, and the Ph.D. degree from École Politechnique Fédérale de Lausanne, in April 2002. From 1999 to 2002, he was with the Mobile Communications Department of Institut Eurécom, Sophia-Antipolis, France, as a Ph.D. student.

In April 2002, he joined the Department of Electrical Engineering of Politecnico di Torino, where he is working as a postdoctoral researcher. His research interests are in the field of signal processing, multiuser detection, space-time coding, sensor networks, and theory of random matrices.

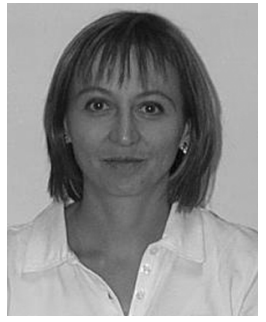

Carla-Fabiana Chiasserini (M'98-SM'09) received the Laurea degree in electrical engineering from the University of Florence, Italy, in 1996. She received the Ph.D. degree from the Politecnico di Torino, Italy, in 1999.

Since then she has been with the Department of Electrical Engineering at the Politecnico di Torino, where she is currently an Associate Professor. From 1998 to 2003 , she worked as a visiting researcher at the University of California at San Diego. Her research interests include architectures, protocols, and performance analysis of wireless networks for integrated multimedia services.

Dr. Chiasserini is a member of the Editorial Board of the Ad Hoc Networks Journal (Elsevier) and has served as an Associate Editor of the IEEE COMMUNICATIONS LETTERS since 2004.

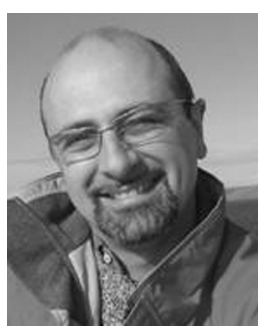

Emanuele Viterbo (M'95-SM'05) was born in Torino, Italy, in 1966. He received the Laurea degree in 1989 and the Ph.D. degree in 1995, both in electrical engineering and both from the Politecnico di Torino, Torino, Italy.

From 1990 to 1992 , he was a patent examiner in the field of dynamic recording and error-control coding with the European Patent Office, The Hague, The Netherlands. Between 1995 and 1997, he held a postdoctoral position in communications techniques over fading channels in the Dipartimento di Elettronica of the Politecnico di Torino. He became Associate Professor at Politecnico di Torino, Dipartimento di Elettronica in 2005, and since November 2006 he has been a Full Professor in the Dipartimento di Elettronica, Informatica e Sistemistica (DEIS), at the Università della Calabria, Italy. In 1993, he was a visiting researcher in the Communications Department of DLR, Oberpfaffenhofen, Germany. In 1994 and 1995, he was visiting the Ècole Nationale Supérieure des Télécommunications (E.N.S.T.), Paris, France. In 1998, he was visiting researcher in the Information Sciences Research Center of AT\&T Research, Florham Park, NJ. In 2003, he was a visiting researcher at the Math Department of EPFL, Lausanne, Switzerland. In 2004, he was a visiting researcher at the Telecommunications Department of UNICAMP, Campinas, Brazil. In 2005, he was a visiting researcher at the ITR of UniSA, Adelaide, Australia. His main research interests are in lattice codes for the Gaussian and fading channels, algebraic coding theory, algebraic space-time coding, digital terrestrial television broadcasting, and digital magnetic recording.

Dr. Viterbo was awarded a NATO Advanced Fellowship in 1997 from the Italian National Research Council. He is Associate Editor of the IEEE TRANSACTIONS ON INFORMATION THEORY, the European Transactions on Telecommunications, and the Journal of Communications and Networks. 